\title{
Taurine in Congestive Heart Failure
}

\section{Farrukh Ahmad, $M D^{1^{*}}$, Nitish Kumar Sharma, $M D^{2}$ and Michelle Hadley, DO, FACC ${ }^{3}$}

${ }^{1}$ Resident, Internal Medicine, Saint Vincent Hospital, Worcester, MA, USA

${ }^{2}$ Cardiology Fellow, Department of Cardiology, Saint Vincent Hospital, Worcester, MA, USA

${ }^{3}$ Department of Cardiology, Saint Vincent Hospital, Worcester, MA, USA

*Corresponding author: Farrukh Ahmad, MD, Resident, Internal Medicine, Saint Vincent Hospital, 123 Summer Street, Worcester, MA 01608, USA, Tel: +1-774-242-0433

\begin{abstract}
Taurine is a ubiquitous amino acid found across the animal kingdom. It is a sulfur-containing amino acid, found in high concentration in the intracellular compartment of excitable tissue, including the myocardium. It functions as an intracellular osmolyte, involved in cell volume regulation. Being a neutral zwitterion, transport of taurine is not accompanied by a change in charge gradient across membranes. This chemical property makes taurine the perfect candidate of cellular osmoregulation. Taurine also regulates sodium and calcium homeostasis, and normal functioning of mitochondria. It has demonstrated ionotropic effects, probably due to its effect on calcium metabolism. Several clinical trials have shown that taurine supplementation improves cardiac performance in those suffering from congestive heart failure. Given its extensive safety profile, taurine supplementation may be beneficial in patients with congestive heart failure.
\end{abstract}

\section{Introduction}

Taurine [1], is a non-proteinogenic sulfur-containing amino acid. Named after the Greek word for Bull, Taurus, Taurine was first isolated from ox bile in 1827 by Friedrich Tiedemann and Leopold Gmelin [2]. Taurine has since been found in most human organs, with the highest concentration in excitable tissue like the heart and brain.

It plays several indispensable roles in normal physiological functions in the human body. Taurine can be considered as a semi-essential amino acid, as it can be synthesized from dietary cysteine. However, diet remains a significant source of taurine. It is considered an essential nutrient in preterm infants, as they have much lower activity of enzymes needed for taurine synthesis. Human breast milk contains about $50 \mathrm{mg} / \mathrm{L}$ [3] of taurine compared to cow milk having $1 \mathrm{mg} / \mathrm{L}$ [4]. Taurine has been an ingredient in commercial infant formula since the 1980s in the United States, with concentration around $10 \mathrm{mg} / \mathrm{L}$.

\section{Taurine metabolism}

Mammalian taurine synthesis occurs in the liver via the cysteine sulfinic acid pathway [BioCyc ID: PWY-5331] from L-cysteine via the action of cysteine dioxygenase (CDO). CDO regulates intracellular cysteine levels, as high levels can be toxic, with low cysteine levels causing degradation of this enzyme and vice versa. The rate limiting step of the taurine synthesis is the enzyme cysteine sulfinic acid decarboxylase (CSAD). Certain animals like mice have a high expression of this enzyme and can synthesize taurine in sufficient quantities provided adequate cysteine in diet. Carnivorous animals like cats and dogs have a lower expression of CSAD and thus require taurine from their diet. Taurine depletion results in cardiomyopathy in these animals.

Diet is the main source of taurine in humans with meat, poultry and especially seafood being rich sources. Low taurine intake has been correlated with lower plasma and urine taurine in vegans [5]. Total body taurine is regulated via the kidney, with increased taurine excretion under high exogenous taurine loads. Taurine supplements increase tissue taurine concentration [6]. It is estimated that a $70 \mathrm{~kg}$ Human contains about $5-7 \mathrm{~g}$ of taurine [7].

Taurine is absorbed from the ileum via Tau-T transport protein [SLC6A6 gene, $2 \mathrm{Na}^{+}: 1$ taurine: $1 \mathrm{Cl}^{-}$, 
with high oral bioavailability. After oral administration plasma levels peak in about 1.5 hours and return to baseline by 8 hours. Cellular taurine pool is maintained by uptake via Tau-T transport protein and efflux via osmosentive channels.

Radiolabeled tracer studies have shown that taurine is present in two pools, an extracellular smaller pool and a much larger intracellular pool [8]. Taurine has a volume of distribution of around 40 L [9]. Extracellular taurine represents $2 \%$ of total body taurine and has a much more rapid exchange rate (t1/2-0.1 hr). This compared to a much larger intracellular pool of taurine with a much slower turnover rate (t1/2-70 hours). Therefore, most of the total body taurine is present in the intracellular compartment.

The role of taurine in mammalian physiology is diverse, but the most apparent one being cell volume regulation. Cells maintain functionality within a narrow range of cell volumes. Osmotic and metabolic factors can alter cell volumes. To maintain volume, cells must alter cytosolic osmolarity by moving osmolytes like taurine, glutamate, potassium and chloride ions in and out of the cells. Taurine is a neutral zwitterion and thus its movement does not alter membrane potentials, unlike movement of other major osmolytes like potassium and chloride ions.

To understand the fascinating role of taurine in cell volume regulation [10], consider the following scenarios-

- Under metabolic stress, including ischemia, decrease in supply of ATP causes failure of $\mathrm{Na}-\mathrm{K}$ ATPase, thus increasing intracellular sodium. This causes fluid shift into the cells leading to cell swelling. Under cell volume regulation, in response to this swelling, cells actively lose osmolytes like taurine into the extracellular fluid resulting in intracellular fluid loss thus mitigating the swelling [11].

- A similar response of taurine loss is seen under hypotonic stress. Hypotonic conditions cause cell swelling by movement of fluid into the cell. Cells by losing taurine cause faster equilibration of the two compartments to control cell swelling. This is termed as regulatory volume decrease (RVD) $[12,13]$.

- The reverse happens under hypertonic stress, such as dehydration. A hypertonic stress will cause fluid shift from the intracellular compartment into the extracellular compartment, leading to cell shrinkage. In response to this, cells increase the expression of taurine transporters, and the uptake of taurine increases cell osmolarity, holding fluid back, thus maintaining cell volume $[14,15]$.

Thus by moving inert osmolytes like taurine, cells are able to regulate fluid movements and buffer cell volume. Systemic effects of this phenomenon have not been well studied.

Apart from Cell volume regulation taurine plays various physiological roles and demonstrates pleitropic pharmacological effects. Bile acid conjugation, mitochondrial function, and a potent antioxidant. It is an effective scavenger of Hypochlorous acid generated under scenarios of metabolic stress $[16,17]$.

\section{Taurine and the Heart}

Taurine is an essential nutrient in certain mammals, including the cat family. The essential role of Taurine in mammalian cardiac physiology is evident because taurine depletion in cats, dogs, and foxes causes the rapid development of dilated cardiomyopathy, which is reversible on introduction of taurine [18].

Mice have the capacity to synthesize sufficient taurine de-novo due to higher expression of CSD, and thus to study taurine depletion in mice, taurine transporter knockout mice have been generated. These mice can synthesize taurine normally, but are unable to concentrate it into the cells. These mice develop dilated cardiomyopathy at 9 months of age, and examination of heart tissue show ventricular remodeling with significant ultrastructural damage of myofilaments and mitochondria. The knockout mice also lose weight, have poor exercise tolerance and have undetectable levels of taurine in heart and skeletal muscle [19].

Taurine has demonstrated several effects that may be beneficial in congestive heart failure.

\section{lonotropic effects of taurine}

Taurine depleted cats develop systolic dysfunction, with a decrease in rate of pressure generation and an impaired relaxation time. Taurine deficient myocardium has a lower calcium load, increased troponin I phosphorylation, and decreased excitation-contraction coupling, leading to decreased myocardial contractility. These effects are reversed by taurine administration [20].

The mechanism of inotropic effect of taurine is thought to be via its alteration in calcium metabolism [21]. Acute administration of taurine increases cytosolic calcium [22]. An increase in extracellular taurine causes an increase in taurine uptake by myocardium via the $\mathrm{Na}$-Taurine, this increase in intracellular $\mathrm{Na}$, causes influx of cytosolic $\mathrm{Ca}^{2+}$ via $\mathrm{Na}-\mathrm{Ca}$ exchanger, similar to digoxin $[23,24]$. It also increases $\mathrm{Ca}^{2+}$ sensitivity of contractile proteins thus increasing the rate of tension development [25]. Chronic administration of taurine by phosphorylating phospholamban, a protein on the SR, disinhibits sarcoplasmic reticulum Ca-ATPase, and increases intra-sarcoplasmic calcium concentration. This also increases relaxation time, and improves diastolic function [26]. The calcium sensitizing effect of taurine 
is the probable explanation for the inotropic effect of taurine.

\section{Mitochondrial functioning}

Taurine is intricately involved in mitochondrial functioning [27]. It functions as a chemical buffer to maintain the $\mathrm{pH}$ of the mitochondrial matrix. The electron transport chain functions by pumping hydrogen ions out of the matrix into the periplasmic space to generate a concentration gradient, which is essential for the functioning of ATPase. ATPase then allows hydrogen ions to move down this gradient, converting ADP to ATP. Mitochondrial matrix has been shown to have a $\mathrm{pH}$ of around 8 [28], closely coinciding with pKa of the amino group of Taurine at 8.6 at body temperature. Therefore, Taurine is a suitable buffer in the mitochondrial matrix and is shown to be present in high concentrations in this space [28]. Under high energy demands, this buffering capacity of Taurine becomes more apparent, as TauT KO mice demonstrate decreased exercise capacity [29]. Additionally, this buffering capacity is also essential for the citric acid cycle, as most of the enzymes of the cycle function optimally at higher $\mathrm{pH}$ [30]. Congestive heart failure decreases myocardial mechanical efficiency [31], and optimizing mitochondrial function should, in theory, improve energy metabolism.

\section{Diuretic effects of taurine}

Diuretic therapy constitutes an important element in the management of heart failure. Taurine administration in animal models has shown a diuretic effect by increasing sodium excretion [32]. In patients suffering from cirrhosis intravenous administration of taurine was able to transiently increase diuresis and natriuresis by suppressing the renin-angiotensin aldosterone system [33]. Taurine has also been shown to suppress vasopressin and promote free water loss [34]. This diuretic effect of taurine may explain some of the beneficial effects seen in heart failure.

\section{Taurine and angiotensin II signaling}

ACE inhibitors are among the first line agents in the management of CHF. They have shown mortality benefits in congestive heart failure.

ACE inhibitors demonstrate these benefits by suppressing the pathological over activation of RAAS thereby augmenting cardiac remodeling and fibrosis, generally seen in untreated CHF. As above, taurine administration has been shown to suppress renin via its effects of renal salt delivery. In vitro studies of rat cardiomyocytes have shown that taurine also prevents angiotensin $\mathrm{II}$ induced $[\mathrm{H}] 3$-phenylalanine and $[\mathrm{H}] 3$ thymidine uptake, demonstrating that taurine is preventing angiotensin induced protein synthesis and DNA replication. Taurine has also shown to downregulate angiotensin 2 receptors, in cardiac myocytes [35].

Angiotensin II is also released by cardiac myocytes in a paracrine fashion as a response to activation of cellular stretch receptors. Taurine plays an important role in cell volume regulation, and efflux of taurine has been demonstrated in cardiac myocytes as a response to cell swelling. This loss of taurine better controls cell swelling and prevents activation of stretch receptors. Thus suppressing Angiotensin signaling by taurine may have similar benefits as ACE inhibitors, in CHF.

\section{Sympatholytic effects of taurine}

One of the hallmarks of congestive heart failure is increased sympathetic activity, which has been shown to accelerate ventricular remodelling and vascular resistance. Taurine has been shown to prevent isoprenaline-induced cardiotoxicity in chick hearts [36]. Taurine also suppresses downstream effects of norepinephrine in the heart, as seen by decreased norepinephrine- induced activation of calpain, a calcium-dependent protease that contributes to cardiomyocyte injury [37]. In another study, isolated mice mesenteric arteries, vasoconstriction induced by norepinephrine was significantly decreased by incubation in taurine containing media [38].

\section{Taurine as an anti-hypertensive}

Taurine administration has shown to improve blood pressure control in pre-hypertensive individuals [39]. In a 2016 study, Sun, et al. randomly assigned 120 prehypertensives patients to receive $1.6 \mathrm{~g}$ of taurine per day or placebo for 12 weeks. Taurine supplementation significantly decreased the clinic and 24-hour ambulatory BPs, especially in those with high-normal BP. Mean clinic systolic BP reduction for taurine/placebo was $7.2 / 2.6 \mathrm{mmHg}$, and diastolic BP was $4.7 / 1.3 \mathrm{mmHg}$. The mean ambulatory systolic BP reduction for taurine/ placebo was $3.8 / 0.3 \mathrm{mmHg}$, and diastolic BP was 3.5/0.6 mm Hg. Taurine supplementation has shown to improve endothelium dependent vasodilation and increase responsiveness to Nitric oxide [40]. Taurine also increases plasma $\mathrm{H} 2 \mathrm{~S}$, which has vasodilatory effects similar to NO.

Diuretics, ACE inhibitors and sympatholytics are the mainstay of CHF management. Taurine demonstrates these effects at clinically relevant doses, along with inotropic and cardioprotective effects [11].

\section{Review of Relevant Literature}

Taurine supplementation in clinical trials on patients with congestive heart failure has demonstrated significant improvements in exercise capacity and cardiac functioning (Table 1).

In a double-blind crossover trial involving 14 patients with CHF published in 1985, Taurine supplementation showed improvements in the treatment group, as determined by a heart failure score [41]. In this study, the researchers supplemented $6 \mathrm{~g} /$ day of Taurine for 4 weeks, with a 2 week wash-out period before 


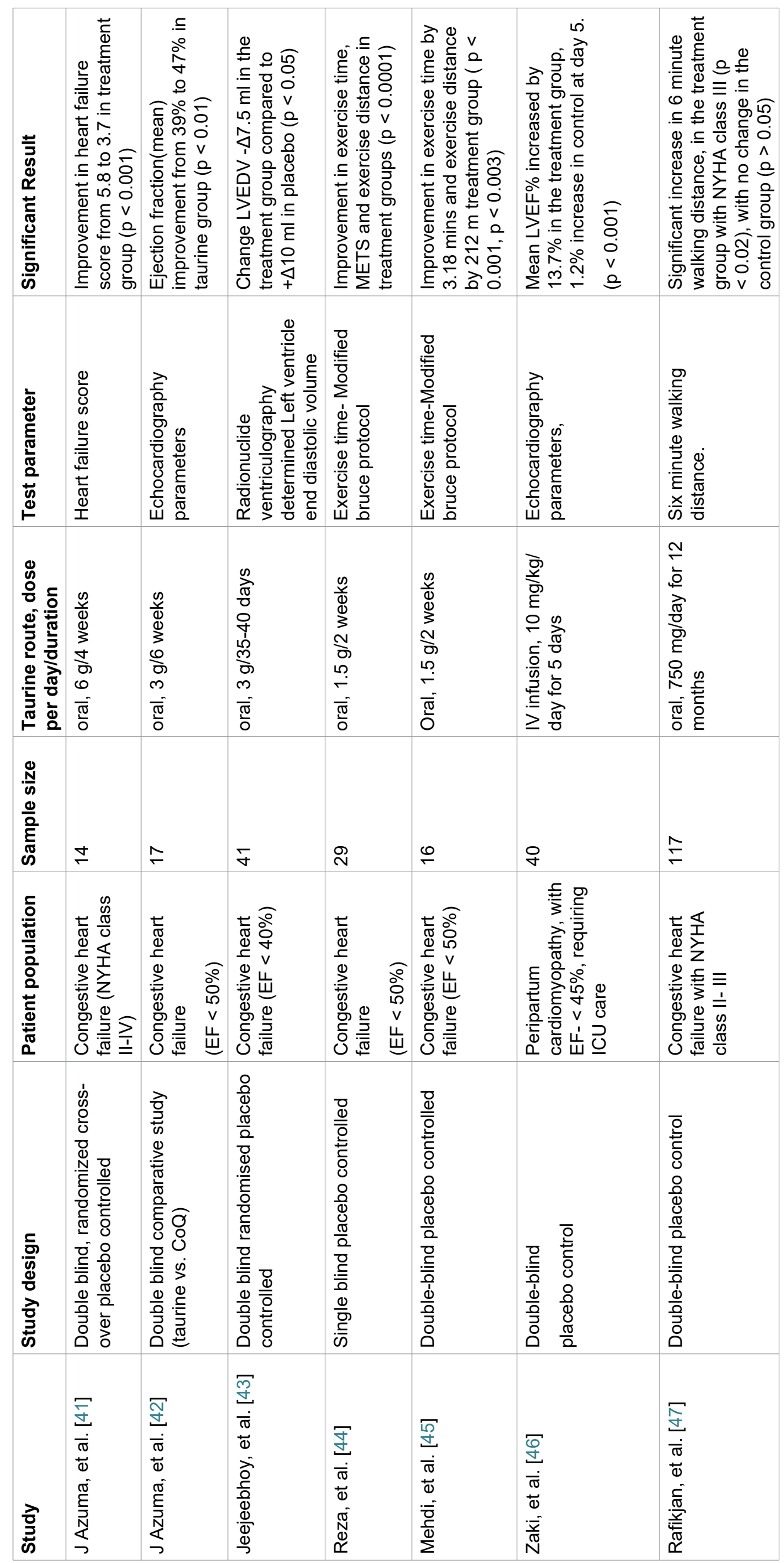


crossing over the groups. They used clinico-radiographic parameters to assess the severity of heart failure and changes post-treatment. Twelve of 14 patients in the treatment group demonstrated improvements compared to $3 / 14$ in the placebo group. The same group published another double-blind placebo-controlled randomized controlled trial in 1993, comparing the effect of Taurine and CoQ in CHF. A total sample size of 17 patients with ejection fraction $<50 \%, 10$ patients in the taurine group receiving $3 \mathrm{~g}$ of Taurine per day for 6 weeks. A significant improvement in ejection fraction and cardiac output was observed on echocardiography in the Taurine group compared to patients supplemented with $\mathrm{CoQ}$ [42].

Jeejeebhoy, et al. conducted a double-blind, randomized controlled trial on 41 patients with an ejection fraction of $<40 \%$ who were scheduled for aortocoronary bypass. With supplementation of amino acid drink fortified with Taurine, $\mathrm{CoQ}$, and carnitine, the researcher demonstrated a decrease in left ventricular end diastolic volume in the treatment group compared to the placebo group. They also showed an increase in myocardial taurine content post-supplementation via a myocardial biopsy [43].

A single-blind placebo-controlled trial published in 2011 by M Reza, et al. studied 29 patients with a left ventricular ejection fraction (LVEF) less than $50 \%$ and NYHA class III-IV symptoms. Fifteen patients in the treatment received $1.5 \mathrm{~g}$ of Taurine per day for 2 weeks in addition to the standard of care heart failure therapy. They used exercise time, METS and exercised distance as their primary parameters. The study demonstrated significant improvement in the exercise time and distance with higher METs achievement in the group receiving Taurine supplementation [44].

Ahmadian $\mathrm{M}$, et al. in a double-blind, randomized placebo controlled trial involving16 patients with heart failure, studied the effect of Taurine supplementation of $1.5 \mathrm{gm}$ per day for 2 weeks. Using the modified bruce protocol to assess the functional capacity, the study demonstrated a significant increase in METS and distance traveled in the treatment group, which was accompanied by a decrease in rate-pressure product [45].

In a double blind, randomized placebo controlled trial involving 40 patients with peripartum cardiomyopathy requiring ICU level of care Zaki, et al. the treatment group received continuous infusion of taurine and the placebo group received a matched volume of isotonic saline. At five days of treatment seventeen patients $(85 \%)$ in the taurine group showed improvement of the NYHA functional class at day 5. In contrast, only two patients $(10 \%)$ in the control group showed improvement of NYHA functional class at day 5. They also demonstrated significant improvement in ejection fraction and left ventricular systolic diameter, not seen in the control group [46].
In another randomized control trial, by Rafikjan, et al. (2021), 117 patients with Congestive heart failure with NYHA class II and above, were randomized into the treatment group receiving $750 \mathrm{mg}$ of Taurine for 12 months, and a placebo group. Both groups were continued on standard goal directed therapy. In the group of patients taking taurine, by the end of the observation period, a decrease in the functional class of CHF was noted in 36 out of 64 patients (56\%) [47].

\section{Safety of Taurine}

Taurine is a dietary amino acid, and is a common ingredient in several commercially available energy drinks, and is available as an over-the-counter supplement in most countries. After oral administration, the circulating levels of Taurine peak at 2-3 hours and return to baseline by 8 hours. Total body taurine is regulated by renal excretion, and high doses of Taurine have not shown any adverse effects [9]. Pharmacologic doses of Taurine used intravenously and orally were associated with no serious adverse effects. Taurine at 5 $\mathrm{g}$ intravenous doses in a clinical trial to assess its effect on reperfusion injury [48], and 2-6 g/day orally for 6 months in children with fatty liver [49] produced no toxicity.

Taurine has received GRAS (Generally recognised as Safe) status by FDA [GRN No. 586].

It must be kept in mind that rapid infusion of taurine at high doses can in theory increase cytosolic calcium enough to cause cytosolic calcium overload, this is particularly important under conditions of acute $\mathrm{MI}$, when the myocardium is vulnerable. Taurine should be avoided during myocardial ischemia.

Taurine has also been shown to build up in the blood and tissue of patients with end stage renal disease [ESRD], with side effects, including dizziness and drowsiness. The symptoms resolved on withdrawal of the drug. Hence, care should be taken when administering taurine in patients with ESRD [50].

In patients on anti-arrhythmic medication, care should be taken, as taurine itself poses antiarrhythmic properties [51,52] and may precipitate bradyarrhythmias.

\section{Authors Recommendations}

Taurine is a dietary nutrient found in meats, poultry and seafood, with scallops being the richest source. It can be synthesized by humans, at least enough to prevent overt signs of deficiency in healthy individuals. As seen above taurine supplementation of doses from $1 \mathrm{~g} /$ day to $5 \mathrm{~g} /$ day demonstrated benefits in congestive heart failure.

Taurine has a slow intracellular turnover rate, and over the course of days to weeks of therapy, tissue taurine levels gradually build up, until reaching a steady 
state. Given the above facts, taurine can be administered at doses of $1 \mathrm{~g}-2 \mathrm{~g}(15-20 \mathrm{mg} / \mathrm{kg})$ once a day orally, in patients with congestive heart failure, causing gradual tissue build up over the course of a few weeks. Patients should be monitored for signs for drowsiness and lethargy. Rapid loading of taurine ( $>5 \mathrm{~g} /$ day) should be avoided, especially in situations of myocardial ischemia, as it offers no added advantage and may cause harm.

\section{Summary}

Taurine is a dietary amino acid with several crucial physiological functions. It is actively concentrated in the intracellular compartment by secondary active transport via the Taurine transporter (TauT). It plays a significant role as an intracellular osmolyte and allows for appropriate cell volume regulation in response to osmotic stress. Taurine also modulates various ion channels in the myocardium and has been shown to increase intracellular calcium concentration by increasing $\mathrm{Na}-\mathrm{Ca}$ exchange activity, an action that is similar to digoxin. It also increases the uptake of calcium ions into the sarcoplasmic reticulum, which improves excitation-contraction coupling and diastolic function. Taurine acts as a mitochondrial $\mathrm{pH}$ buffer, allowing for the normal functioning of the electron transport chain and citric acid cycle, especially under high energy demand states. Along with an inotropic effect, taurine also functions as a diuretic, blocks angiotensin signaling and inhibits Vasopressin. Taurine supplementation has been shown to increase aerobic performance in healthy individuals. Given the beneficial effects of Taurine supplementation in myocardial performance in congestive heart failure (CHF), Taurine supplementation is included as a standard of treatment in the management of congestive heart failure in Japan [53]. Patients with congestive heart failure are likely taurine depleted due to myocardial dysfunction and dietary modification. Repleting Taurine to physiological levels via supplementation has been shown to improve cardiac function.

Given its extensive safety profile and potential to improve quality of life in patients with congestive heart failure, Taurine may serve as a therapeutic modality in the management of heart failure. However, extensive scale and longer-term clinical trials are needed to confirm these findings before taurine gets approval from the FDA for use in congestive heart failure.

\section{References}

1. (2021) National Center for Biotechnology Information. PubChem Compound Summary for CID 1123, Taurine.

2. Tiedemann F, Gmelin L (1827) Einige neue Bestandtheile der Galle des Ochsen. Ann Phys 85: 326-337.

3. Heird WC (2004) Taurine in neonatal nutrition--revisited. Arch Dis Child Fetal Neonatal Ed 89: F473-F474.

4. Klein CJ (2002) Nutrient requirements for preterm infant formulas. J Nutr 132: 1395S-1577S.
5. Laidlaw SA, Shultz TD, Cecchino JT, Kopple JD (1988) Plasma and urine taurine levels in vegans. Am J Clin Nutr 47: $660-663$

6. De Luca A, Pierno S, Camerino DC (2015) Taurine: The appeal of a safe amino acid for skeletal muscle disorders. J Transl Med 13: 243.

7. Huxtable RJ (1992) Physiological actions of taurine. Physiol Rev 72: 101-163.

8. Sturman JA, Hepner GW, Hofmann AF, Thomas PJ (1975) Metabolism of [35S] taurine in man. J Nutr 105: 1206-1214.

9. Ghandforoush-Sattari M, Mashayekhi S, Krishna CV, Thompson JP, Routledge PA (2010) Pharmacokinetics of oral taurine in healthy volunteers. J Amino Acids 2010: 346237.

10. Hoffmann EK, Dunham PB (1995) Membrane mechanisms and intracellular signalling in cell volume regulation. Int Rev Cytol 161: 173-262.

11. Schaffer SW, Jong CJ, Ito T, Azuma J (2014) Effect of taurine on ischemia-reperfusion injury. Amino Acids 46: 21 30.

12. Okada Y, Maeno E, Shimizu T, Dezaki K, Wang J, et al. (2001) Receptor-mediated control of regulatory volume decrease (RVD) and apoptotic volume decrease (AVD). J Physiol 532: 3-16.

13. Guizouarn H, Motais R, Garcia-Romeu F, Borgese F (2000) Cell volume regulation: The role of taurine loss in maintaining membrane potential and cell $\mathrm{pH}$. J Physiol 523 Pt 1: 147-154.

14. Trachtman H, Futterweit S, del Pizzo R (1992) Taurine and osmoregulation. IV. Cerebral taurine transport is increased in rats with hypernatremic dehydration. Pediatr Res 32: 118-124.

15. Baliou S, Kyriakopoulos AM, Goulielmaki M, Panayiotidis MI, Spandidos DA, et al. (2020) Significance of taurine transporter (TauT) in homeostasis and its layers of regulation (Review). Mol Med Rep 22: 2163-2173.

16. Lambert IH, Kristensen DM, Holm JB, Mortensen OH (2015) Physiological role of taurine--from organism to organelle. Acta Physiol (Oxf) 213: 191-212.

17. Kearns S, Dawson R Jr (2000) Cytoprotective effect of taurine against hypochlorous acid toxicity to PC12 cells. Adv Exp Med Biol 483: 563-570.

18. Knopf K, Sturman JA, Armstrong M, Hayes KC (1978) Taurine: An essential nutrient for the cat. J Nutr 108: 773778.

19. Ito T, Oishi S, Takai M, Kimura Y, Uozumi Y, et al. (2010) Cardiac and skeletal muscle abnormality in taurine transporter-knockout mice. Journal of Biomedical Science 17: $1-5$.

20. Novotny MJ, Hogan PM, Paley DM, Adams HR (1991) Systolic and diastolic dysfunction of the left ventricle induced by dietary taurine deficiency in cats. Am J Physiol 261: H121-A127.

21. Franconi $F$, Stendardi I, Matucci R, Failli $P$, Bennardini $F$, et al. (1984) Inotropic effect of taurine in guinea-pig ventricular strips. Eur J Pharmacol 102: 511-514.

22. Bkaily G, Jaalouk D, Haddad G, Gros-Louis N, Simaan M, et al. (1997) Modulation of cytosolic and nuclear Ca2+ and $\mathrm{Na}+$ transport by taurine in heart cells. Mol Cell Biochem 170: 1-8.

23. Satoh $H$, Sperelakis N (1998) Review of some actions of 
taurine on ion channels of cardiac muscle cells and others. Gen Pharmacol 30: 451-463.

24. Bkaily G, Jaalouk D, Sader $S$, Shbaklo $H$, Pothier $P$, et al. (1998) Taurine indirectly increases [Ca]i by inducing $\mathrm{Ca} 2+$ influx through the $\mathrm{Na}(+)-\mathrm{Ca} 2+$ exchanger. Mol Cell Biochem 188: 187-197.

25. Galler S, Hutzler C, Haller T (1990) Effects of taurine on Ca2 (+)-dependent force development of skinned muscle fibre preparations. J Exp Biol 152: 255-264.

26. Ramila KC, Jong CJ, Pastukh V, Ito T, Azuma J, et al. (2015) Role of protein phosphorylation in excitation-contraction coupling in taurine deficient hearts. Am J Physiol Heart Circ Physiol 1308: H232-H239.

27. Hansen SH, Andersen ML, Cornett C, Gradinaru R, Grunnet $\mathrm{N}$ (2010) A role for taurine in mitochondrial function. $J$ Biomed Sci 17: S23.

28. Porcelli AM, Ghelli A, Zanna C, Pinton P, Rizzuto R, et al. (2005) $\mathrm{pH}$ difference across the outer mitochondrial membrane measured with a green fluorescent protein mutant. Biochem Biophys Res Commun 326: 799-804.

29. Warskulat U, Flögel U, Jacoby C, Hartwig HG, Thewissen $M$, et al. (2004) Taurine transporter knockout depletes muscle taurine levels and results in severe skeletal muscle impairment but leaves cardiac function uncompromised. FASEB J 18: 577-579.

30. Ghisla S, Thorpe C (2004) Acyl-CoA dehydrogenases. A mechanistic overview. Eur J Biochem 271: 494-508.

31. Baxley WA, Dodge HT, Rackley CE, Sandler H, Pugh D (1977) Left ventricular mechanical efficiency in man with heart disease. Circulation 55: 564-568.

32. Mozaffari MS (2003) Taurine modulation of renal excretory function. TJPS 16: 83-90.

33. Gentile S, Bologna E, Terracina D, Angelico M (1994) Taurine-induced diuresis and natriuresis in cirrhotic patients with ascites. Life Sci 54: 1585-1593.

34. Mozaffari MS, Schaffer D (2001) Taurine modulates arginine vasopressin-mediated regulation of renal function. J Cardiovasc Pharmacol 37: 742-750.

35. Li C, Cao L, Zeng Q, Liu X, Zhang Y, et al. (2005) Taurine may prevent diabetic rats from developing cardiomyopathy also by downregulating angiotensin II type2 receptor expression. Cardiovasc Drugs Ther 19: 105-112.

36. Ohta H, Azuma J, Onishi S, Awata N, Takihara K, et al. (1986) Protective effect of taurine against isoprenalineinduced myocardial damage. Basic Res Cardiol 81: 473481

37. Li Y, Arnold JM, Pampillo M, Babwah AV, Peng T (2009) Taurine prevents cardiomyocyte death by inhibiting NADPH oxidase-mediated calpain activation. Free Radic Biol Med 46: 51-61.

38. Li N, Sawamura M, Nara Y, Ikeda K, Yamori Y (1996) Direct inhibitory effects of taurine on norepinephrineinduced contraction in mesenteric artery of stroke-prone spontaneously hypertensive rats. Adv Exp Med Biol 403: 257-262.
39. Waldron M, Patterson SD, Tallent J, Jeffries O (2018) The effects of oral taurine on resting blood pressure in humans: A meta-analysis. Curr Hypertens Rep 20: 81.

40. Sun Q, Wang B, Li Y, Sun F, Li P, et al. (2016) Taurine supplementation lowers blood pressure and improves vascular function in prehypertension: Randomized, doubleblind, placebo-controlled study. Hypertension 67: 541-549.

41. Azuma J, Sawamura A, Awata N, Ohta H, Hamaguchi T, et al. (1985) Therapeutic effect of taurine in congestive heart failure: A double-blind crossover trial. Clin Cardiol 8: 276282.

42. Azuma J, Sawamura A, Awata N (1992) Usefulness of taurine in chronic congestive heart failure and its prospective application. Jpn Circ J 56: 95-99.

43. Jeejeebhoy F, Keith M, Freeman M, Barr A, McCall M, et al. (2002) Nutritional supplementation with MyoVive repletes essential cardiac myocyte nutrients and reduces left ventricular size in patients with left ventricular dysfunction. Am Heart J 143: 1092-1100.

44. Beyranvand MR, Khalafi MK, Roshan VD, Choobineh S, Parsa SA, et al. (2011) Effect of taurine supplementation on exercise capacity of patients with heart failure. J Cardiol 57: 333-337.

45. Ahmadian M, Dabidi Roshan V, Ashourpore E (2017) Taurine supplementation improves functional capacity, myocardial oxygen consumption, and electrical activity in heart failure. J Diet Suppl 14: 422-432.

46. Zaki HV, Sweed MS, Ali RM, Abdelhafeez MA (2021) Taurine as an adjunct therapy for early left ventricular recovery in peripartum cardiomyopathy. Journal of Obstetric Anaesthesia and Critical Care 11: 9.

47. Yuldashev R, Ibragimjon I, Yunusova Z, Dauletbaev $B$, Abdurahmonov M (2021) The effect of taurine on the clinical course and mid-term prognosis of chronic heart failure in patients with ischemic heart disease. Annals of the Romanian Society for Cell Biology 25: 746-752.

48. Milei J, Ferreira R, Llesuy S, Forcada P, Covarrubias J, et al. (1992) Reduction of reperfusion injury with preoperative rapid intravenous infusion of taurine during myocardial revascularization. Am Heart J 123: 339-345.

49. Obinata K, Maruyama T, Hayashi M, Watanabe T, Nittono $H$ (1996) Effect of taurine on the fatty liver of children with simple obesity. Adv Exp Med Biol 403: 607-613.

50. Suliman ME, Bárány P, Filho JCD, Lindholm B, Bergström J (2002) Accumulation of taurine in patients with renal failure. Nephrology Dialysis Transplantation 17: 528-529.

51. Eby G, Halcomb WW (2006) Elimination of cardiac arrhythmias using oral taurine with l-arginine with case histories: Hypothesis for nitric oxide stabilization of the sinus node. Med Hypotheses 67: 1200-1204.

52. Wang GX, Duan J, Zhou S, Li P, Kang Y (1992) Antiarrhythmic action of taurine. Adv Exp Med Biol 315: 187-192.

53. Schaffer S, Kim HW (2018) Effects and mechanisms of taurine as a therapeutic agent. Biomol Ther (Seoul) 26: 225-241. 